\title{
SYNCHRONOUS PRIMARY MALIGNANCY OF ENDOMETRIUM AND CERVIX
}

\author{
Mangirish Anil Kenkre1, Guruprasad Pednekar², Ajit Nagarsenkar³, Sharvani Pai ${ }^{4}$ \\ ${ }^{1}$ Senior Resident, Department of Obstetrics and Gynaecology, Goa Medical College. \\ 2Professor \& Head, Department of Obstetrics and Gynaecology, Goa Medical College. \\ ${ }_{3}^{3}$ Associate Professor, Department of Obstetrics and Gynaecology, Goa Medical College. \\ 4 Intern, Department of Obstetrics and Gynaecology, Goa Medical College.
}

\begin{abstract}
BACKGROUND

Synchronous primary malignancies of the female genital system are a very rare occurrence. A 67-year female presenting with postmenopausal bleeding with evidence of hyperplastic endometrium of 13 millimetres on MRI scan. An endometrial biopsy revealed Adenocarcinoma of Endometrium. The patient was subjected to staging laparotomy, which revealed stage IA Adenocarcinoma of the Endometrium and Squamous Cell Carcinoma in Situ of the Cervix.
\end{abstract}

\section{KEYWORDS}

Synchronous Primary Malignancy, Endometrium, Cervix.

HOW TO CITE THIS ARTICLE: Kenkre MA, Pednekar G, Nagarsenkar A, et al. Synchronous primary malignancy of endometrium and cervix. J. Evolution Med. Dent. Sci. 2016;5(31):1687-1688, DOI: 10.14260/jemds/2016/397

\section{INTRODUCTION}

Synchronous primary malignancies of the female genital system are a rare occurrence. Amongst the reported cases, most frequent associations are between ovary and endometrium. Such cases pose a dilemma to the treating gynaecologist with regards to aetiology, treating modalities and prognosis. Difficulties in differentiating the two malignancies and excluding metastasis also poses a challenge. We report a case of synchronous adenocarcinoma of endometrium and squamous cell carcinoma in a 67-year-old postmenopausal woman.

\section{CASE REPORT}

A 67 years old hypertensive woman, menopausal since the last 20 years presented in the outpatient department on August 7th, 2014 with one episode of postmenopausal bleeding. She has had previous four vaginal deliveries, the last being 38 years ago. As regards to her previous menstrual cycles were regular.

On examination, her vital parameters were stable and the patient was averagely built. Her abdomen was soft without any signs of ascites or organomegaly. On per speculum examination, the cervix and vagina were healthy. On vaginal examination her uterus was of normal size, anteverted without any palpable mass in the either fornices.

Her pelvic ultrasound revealed a hypertrophic endometrium with endometrial thickness of 8 millimetres. Subsequently, an endometrial sampling and Papanicolaou smear test of cervix was done on outpatient basis. Papanicolaou smear test did not reveal any evidence of malignancy of the cervix. However, her endometrial sampling revealed adenocarcinoma of the endometrium.

Financial or Other, Competing Interest: None.

Submission 04-03-2016, Peer Review 23-03-2016,

Acceptance 28-03-2016, Published 18-04-2016.

Corresponding Author:

Dr. Mangirish Anil Kenkre,

House No. 124,

Next to Datta Temple,

Dattawadi, Mapusa,

Bardez Goa-403507.

E-mail: mangirish.kenkre@gmail.com

DOI: $10.14260 /$ jemds $/ 2016 / 397$
Patient was then subjected to an abdomino-pelvic MRI scan, which confirmed the findings of hypertrophic endometrium with an endometrial thickness of 13 millimetres with cystic areas within endometrium. There was evidence of ill-defined areas of endomyometrial junction in the posterior wall of the uterus. Cervix appeared normal on MRI scan. There was no significant pelvic lymphadenopathy.

Her preoperative haematological and radiological investigations were normal and patient was subjected to a staging laparotomy, wherein an extrafascial total abdominal hysterectomy with bilateral salpingo-oophorectomy was done on. Intraoperatively, her uterus was of normal size measuring $8 \times 5 \times 3$ centimetres, both ovaries and fallopian tubes appeared healthy with cervix looking grossly normal. There was no free fluid in pelvis nor was there any evidence of pelvic lymphadenopathy. Under surface of the diaphragm, liver and the omentum were grossly normal. Patient was stable intraoperatively and postoperatively.

On histopathological examination of the specimen, cut section of the uterus revealed papillary projections in the endometrial cavity over an area of 4 centimetres not reaching up to the uterocervical junction. Grossly, there was no myometrial invasion and cervix and the ovaries did not reveal any significant pathology.

However, on microscopic examination it was seen that the endometrium had a small focus of moderately differentiated adenocarcinoma without myometrial invasion, while the rest of the endometrium revealed cystic atrophy. Microscopic examination of the cervix revealed squamous cell carcinoma in situ.

A diagnosis of endometrial adenocarcinoma stage IA with squamous cell carcinoma in situ of cervix was established in the above 67-year-old postmenopausal patient with hypertension.

\section{DISCUSSION}

Synchronous primary malignancies of the female genital system are defined as occurrence of two or more tumours in a patient closely in time. ${ }^{1}$ They should be differentiated from metastasis from a primary source. The different histopathological picture and occurrence of low histological grade and early stage give an indication that they may have 
multifocal origin rather than metastasis as in our case. ${ }^{2}$ The occurrence of synchronous primary tumours of the female genital system is a rare phenomenon with an incidence of only 0.7 to $1.8 \% .^{3}$ Most frequent amongst them are concurrent presence of endometrial and ovarian malignancies. ${ }^{3}$ The pathogenesis of synchronous malignancies is still a mystery. However, the theory of secondary Mullerian system has been promising. According to this theory, the epithelia of the female genital tract simultaneously respond to carcinogenic stimuli.4 The identification of synchronous malignancies plays an important role in the treatment and prognosis of the patient. Surgical staging play an important role, especially in the above mentioned case, as appropriate surgical staging would provide targeted therapy and minimize the adverse effects of overtreatment simultaneously [Chemotherapeutic and radiation toxicity] and the effects of undertreatment [Recurrence]. The prognosis for a patient with synchronous gynaecologic malignancies does not seem to be worse. ${ }^{5}$

\section{REFERENCES}

1. Woodruff JD, Solomon D, Sullivant H. Multifocal disease in the upper genital tract. Obstet Gynaecol 1985;65:6958.

2. Matloch DL, Salem FA, Charles EH, et al. Synchronous multiple primary neoplasms of the upper female genital tract. Gynaecol Oncol 1982;13(2):271-7.

3. Eisner RF, Nieberg RK, Berek JS. Synchronous primary neoplasms of the female reproductive tract. Gynaecol Oncol 1989;33(3):335-9.

4. Lauchlan S. The secondary mullerian system. Obstet Gynaecol Surv 1972;27(3):133-46.

5. Lin CK, Yu MH, Chu TW, et al. Synchronous occurrence of primary neoplasms in the uterus with squamous cell carcinoma of the cervix and adenocarcinoma of the endometrium. Taiwan J Obstet Gynaecol 2006;45(4):336-9. 